\title{
Manic episode occurring during investigational treatment with pan-class I phosphoinositide 3-kinase inhibitor in a patient with breast cancer
}

\author{
C. Marchesi ${ }^{1}$ | F. Paraboschi ${ }^{1}$ | V. Lucarini $^{1}$ (I) | C. De Panfilis ${ }^{1}$ | M. Tonna ${ }^{2}$ \\ ${ }^{1}$ Department of Neuroscience, Psychiatry Unit, University of Parma, Parma, Italy \\ ${ }^{2}$ Mental Health Service, Local Service of Health Parma, University Hospital, Parma, Italy \\ Correspondence \\ Valeria Lucarini, University of Parma, Department of Neuroscience, Psychiatry Unit, University Hospital, Braga Building, 43126 Parma, Italy. \\ Email: valerie.lucarini@yahoo.it
}

\section{KEYWORDS}

breast cancer, manic episode, mood disorders, oncology, PI3K inhibitor

\section{1 | INTRODUCTION}

Breast cancer as a life-threatening illness can be related to anxiety, demoralization, or depression, ${ }^{1,2}$ which can occur at any moment of the clinical-diagnostic pathway.

Despite the fact that brain metastases, paraneoplastic syndromes, or pharmacotherapy might induce mood alterations, spontaneous hypomanic and manic episodes usually do not occur in patients with breast cancer.

Concerning mood alterations associated with the administration of antitumor drugs, orally available, pan-class I phosphoinositide 3kinase (PI3K) inhibitors, which exert their activity on breast cancer cells positive for estrogen receptor (ER), particularly in some sub-populations (for instance, some PI3K mutations), ${ }^{3}$ can induce anxiety, euphoria, anhedonia, or depression. ${ }^{4}$

We report a case of a patient with breast cancer and without a familial and personal history of mood or anxiety disorders, who experienced a manic episode at the age of 54 during the treatment with an investigational pan-class I PI3K inhibitor.

A written informed consent was obtained from the patient for the publication of this case report. The local Ethical Committee (Comitato Etico per Parma) approved the double-blind placebo controlled clinical trial in which the patient was enrolled (reference number for the approval, 3553; date of approval, 29/01/2013).

\section{I CASE REPORT}

A 54-year-old woman, with a 4-year history of breast cancer with bone metastases, was treated with an oral pan-class I PI3K inhibitor
(buparlisib-BKM120) in a double-blind placebo-controlled clinical trial carried out at the Medical Oncology Unit of the University Hospital of Parma, Italy.

She was married, and she lived with her husband and with their 31-year-old daughter (with motor disability secondary to a pertussisassociated encephalitis when she was 2 years old). The global functioning was satisfying, and overall the patient adopted good coping strategies for her daughter disability.

She entered the trial on February 28, 2014. No major psychopathological concerns emerged during the first 2 months of treatment, while at the beginning of May she complained tiredness, avolition, and demoralization; therefore, the oncologist suggested to take some days off. When on holidays in Paris, on May 9, she became irritable and verbally aggressive. Few days later, when back home, she was hyperactive, easily distractive, talkative, and agitated. Later on, she became insomniac and developed grandiose self-esteem, satisfying the criteria for a full-blown manic episode.

On May 22, the oncologist referred her to the psychiatry consultation-liaison service, where a diagnosis of a manic episode was made. The patient refused any treatment, and she was sectioned and taken into the acute psychiatric ward. At the first evaluation, she was extremely talkative, with flight of ideas, grandiosity, and high irritability.

The patient was put on Olanzapine $10 \mathrm{mg}$ b.i.d. and Lorazepam $2 \mathrm{mg}$ in the evening both intra-muscular. The blood tests showed a mild elevation in levels of the liver enzymes transaminases (AST $55 \mathrm{U} / \mathrm{L} ; \mathrm{ALT} 61 \mathrm{U} / \mathrm{L}$ ) and lactate dehydrogenase (LDH $710 \mathrm{U} / \mathrm{L}$ ) and low iron levels $(30 \mu \mathrm{g} / \mathrm{dL})$. The neurological examination was unremarkable, and the brain computerized axial tomography (CAT) scan did not show any metastases or acute vascular damage. 
In 1 week, her mood condition rapidly improved; Olanzapine (10 mg b.i.d.) was switched to oral administration, and lorazepam was discontinued. She was discharged on June 7, and she attended the outpatients service with psychiatric visits at nearly monthly intervals. One month after the discharge, she reached criteria for complete remission, and in order to plan the duration of maintenance treatment with antipsychotic, the study allocation has been unblinded.

The patient was in the active drug arm; therefore, the investigational drug was discontinued, and in September, the Olanzapine dose was tapered to $10 \mathrm{mg}$ q.d. because the patient complained sedation and tiredness. After the maintenance of a healthy mental condition, the antipsychotic was discontinued. The patient passed away because of breast cancer disease progression in May 2015.

\section{3 | DISCUSSION}

Acute hypomania or mania does not usually occur in patients with breast cancer. Nevertheless, breast cancer and bipolar disorder (BD) can be associated, because PALB2 and BRCA2 were identified as risk genes for breast cancer and $\mathrm{BD}^{5}$ and cytokines play a role in breast cancer development and progression ${ }^{6}$ and in pathophysiology of BD. ${ }^{7}$

The occurrence of a manic episode could have different underlying mechanism.

First, according to the stress-diathesis hypothesis, the emotional distress caused by a diagnosis of cancer could have triggered the onset of $\mathrm{BD}$ in a vulnerable subject (ie, either a familial history of BD or a previous onset of the mood disorder). However, this does not look the case because a previous episode never occurred, nor a familial vulnerability to BD was found.

Secondly, a manic episode could have been associated with brain metastases, which can be found in approximately $10 \%$ of patients with metastatic breast cancer. This condition might be characterized both by behavioral changes, cognitive impairment, and focal neurological symptoms. In this patient, this possibility was excluded by the normal neurological examination and the negative brain CT scan.

Lastly, the manic episode might have been induced by the pharmacological treatment for the breast cancer. The search for novel agents to ensure efficacy with a good tolerability profile is constant. Breast cancer is frequently associated with the activation of the PI3K/AKT/ mTOR pathway, and its block results in apoptosis and inhibits tumor cell proliferation, angiogenesis, and metastases. ${ }^{8}$ In fact, potent panPI3K inhibitors, which act on all the isoforms of class I PI3K ( $\alpha, \beta, \gamma$ and $\delta$ ), have been shown to be active in preclinical and clinical studies in breast cancer. ${ }^{4}$ Interestingly, the same review ${ }^{4}$ reported that mood alterations, such as anxiety, euphoria, anhedonia, or depression, occurred in $17 \%$ to $45 \%$ of patients treated with this class of drugs. To explain this effect, it has been hypothesized that the dysregulation of the PI3K pathway can be associated with changes in serotonin levels, and, therefore, with mental disturbances such as anxiety and depression. ${ }^{\text {? }}$

Summarizing, even though we cannot exclude other possible causes, the timing of symptom development, the negativity of personal and familial psychiatric history, and the absence of clinical neurological

\section{Key points}

- Breast cancer can induce anxiety, demoralization, or depression.

- Hypomanic and manic episodes are unusual in patients with breast cancer.

- PI3K inhibitors have demonstrated antitumoral effectiveness in breast cancer.

- $\mathrm{PI3K}$ inhibitors can be associated with changes in brain serotonin levels and mental disturbances.

- In this case report, pan-class I PI3K inhibitor administration was associated with the development of a manic episode

findings or CT scan alterations suggest a treatment-related manic episode.

To our knowledge, this is the first report that describes the onset of a manic episode during treatment with a pan-class I PI3K inhibitor, showing that also a severe mood disorder can be induced by this class of drugs. Our observation, along with previous findings of depression and anxiety related to this treatment, ${ }^{9}$ suggest that mental health should be carefully monitored in patients treated with PI3K inhibitors and mild mood alterations should be also recognized and properly treated to prevent the development of severe mood episode.

Isoform-specific PI3K inhibitors are becoming more and more popular, because their specificity offers considerable advantages in terms of both efficacy and safety. Specifically, p110a-specific PI3K inhibitors may have the advantage of a neuron-specific regulation of class I PI3K catalytic subunits that would make these compounds associated with a lower risk of induced-mood disorders, especially when compared with pan-PI3K inhibitors. ${ }^{10}$

The authors declare that they have no competing interests.

\section{ORCID}

V. Lucarini $\odot$ http://orcid.org/0000-0002-3553-8818

\section{REFERENCES}

1. Marchesi C, Maggini C. Socio-demographic and clinical features associated with demoralization in medically ill in-patients. Soc Psychiatry Psychiatr Epidemiol. 2007;42(10):824-829. https://doi.org/10.1007/ s00127-007-0230-z

2. Miovic M, Block S. Psychiatric disorders in advanced cancer. Cancer. 2007;110(8):1665-1676. https://doi.org/10.1002/cncr.22980

3. Yang SX, Polley E, Lipkowitz S. New insights on PI3K/AKT pathway alterations and clinical outcomes in breast cancer. Cancer Treat Rev. 2016;45(Apr):87-96. https://doi.org/10.1016/j.ctrv.2016.03.004

4. Sirohi B, Rastogi S, Dawood S. Buparlisib in breast cancer. Future Oncol. 2015;11(10):1463-1470. https://doi.org/10.2217/fon.15.56

5. Tesli M, Athanasiu L, Mattingsdal M, et al. Association analysis of PALB2 and BRCA2 in bipolar disorder and schizophrenia in a Scandinavian case-control sample. Am J Med Genet B Neuropsychiatr Genet. 2010;153B(7):1276-1282. https://doi.org/10.1002/ajmg.b.31098

6. Esquivel-Velázquez M, Ostoa-Saloma P, Palacios-Arreola MI, NavaCastro KE, Castro JI, Morales-Montor J. The role of cytokines in breast 
cancer development and progression. J Interferon Cytokine Res. 2015;35(1):1-16. https://doi.org/10.1089/jir.2014.0026

7. Anderson G, Maes M. Bipolar disorder: role of immune-inflammatory cytokines, oxidative and nitrosative stress and tryptophan catabolites. Curr Psychiatry Rep. 2015;17(2):8. https://doi.org/10.1007/s11920014-0541-1

8. Courtney KD, Corcoran RB, Engelman JA. The PI3K pathway as drug target in human cancer. J Clin Oncol. 2010;28(6):1075-1083. https:// doi.org/10.1200/JCO.2009.25.3641

9. Ackermann TF, Hortnagl H, Wolfer DP, et al. Phosphatidylinositide dependent kinase deficiency increases anxiety and decreases GABA and serotonin abundance in the amygdala. Cell Physiol Biochem. 2008;22(5-6):735-744. https://doi.org/10.1159/000185557
10. Mayer IA, Abramson V, Formisano L, et al. A Phase lb study of alpelisib (BYL719), a PI3Ka-specific inhibitor, with letrozole in ER+/HER2- metastatic breast cancer. Clin Cancer Res. 2017;23(1):26-34. https://doi. org/10.1158/1078-0432.CCR-16-0134

How to cite this article: Marchesi C, Paraboschi F, Lucarini V, De Panfilis C, Tonna M. Manic episode occurring during investigational treatment with pan-class I phosphoinositide 3-kinase inhibitor in a patient with breast cancer. Psycho-Oncology. 2017;1-3. https://doi.org/10.1002/pon.4550 